\title{
On the fine-scale structure of vector fields convected by a turbulent fluid
}

\author{
By P. G. SAFFMAN \\ Department of Mathematics, King's College, London
}

(Received 30 October 1962 and in revised form 17 April 1963)

This paper is a contribution to the study of statistically homogeneous, dynamically passive vector fields convected by a turbulent fluid and subject to a molecular diffusivity $\lambda$ that is small compared with the kinematic viscosity $\nu$. Two types are considered: the first, denoted by $\mathbf{F}$, has the property that the total flux across a material surface moving with the fluid is conserved if $\lambda=0$ (e.g. magnetic field); and the second, denoted by $\mathbf{G}$, is the gradient of a conserved scalar quantity $\theta$ (e.g. temperature gradient). Attention is focused on small-scale variations with length-scale less than $\left(\nu^{3} / \epsilon\right)^{\frac{\pi}{1}}$. A theory of Batchelor's in terms of Eulerian correlations for the distribution of $\theta$ for the case when $\lambda \ll \nu$ is extended and applied to the vector fields, thereby giving equations for the covariance tensors of $\mathbf{F}$ and $\mathbf{G}$ appropriate for separations less than $\left(y^{3} / \epsilon\right)^{\frac{3}{2}}$. According to these equations, the effect of convection on small-scale components of the fields is to amplify and also to distort by reducing the scale; the ratio of these two effects is measured by a parameter $\sigma$. It is shown that if $\sigma<\frac{5}{2}$, the small-scale structure is stable against perturbations however small $\lambda / \nu$ may be, the amplification being eventually balanced by the dissipation which is increased by the reduction of scale. In the case of the quantity $\mathbf{G}, \sigma=1$. The value of $\sigma$ for the case of $\mathbf{F}$ is not known, but reasons are given for believing that it is less than one, and it is concluded that the behaviour of $\overline{\mathbf{F}^{2}}$ and $\overline{\mathbf{G}^{2}}$ in a field of homogeneous turbulence is qualitatively the same. In particular, $\overline{\mathbf{F}^{2}}$ does not grow indefinitely with time as predicted by previous arguments. The correlation functions for small separations and the corresponding spectrum functions for a statistically steady state are obtained. The relation between this analysis and that for random vector fields in a uniform straining motion of infinite extent is considered in detail, for Pearson has shown that, if the strain is an irrotational distortion, then $\overline{\mathbf{F}^{2}} \rightarrow \infty$ with time. It is shown that this divergence is due to the amplification of components with very small wave-numbers or, equivalently, of very large scale, and it is therefore not considered relevant to a study of homogeneous turbulence.

The particular case of the magnetic field in a good conductor is considered. If the Lorentz forces are unimportant, it is estimated that the magnetic energy of a weak seed field will be in general amplified by the turbulence by a factor lying somewhere between the Reynolds and magnetic Reynolds numbers of the turbulence before ohmic dissipation as increased by the reduction of scale limits the growth, and it is suggested further that the magnetic field will eventually decay to zero in the absence of external electromotive forces. 
In an appendix, the theory is applied tentatively to the turbulent vorticity (which satisfies the same equation as $\mathbf{F}$ if $\lambda=\nu$ ) and an expression for the energy spectrum function for very large wave-numbers is deduced. This is compared with an expression given by Townsend, and is found to have a similar qualitative behaviour but gives values about one-half as large.

\section{Introduction}

Consider a dynamically passive, conserved scalar quantity (like temperature) that satisfies the equation

$$
\frac{\partial \theta}{\partial t}+u_{j} \frac{\partial \theta}{\partial x_{j}}=\lambda \nabla^{2} \theta
$$

where $\mathbf{u}$ is the velocity of the fluid and is independent of $\theta$, and $\lambda$ is the molecular diffusivity. The (incompressible) fluid is supposed in turbulent motion at large Reynolds number, the length scale of the energy-containing eddies being $L$. Batchelor (1959) has discussed with novel methods the statistical properties of those components of the $\theta$-distribution that have a length scale small compared with $L$, for the case when $\lambda \ll \nu$ (the kinematic viscosity of the fluid).

In the present paper, we shall consider the application of these ideas to the small-scale statistical properties of vector fields $\mathbf{F}$ and $\mathbf{G}$ which satisfy

and

$$
\begin{array}{ll}
\frac{\partial F_{i}}{\partial t}+u_{j} \frac{\partial F_{i}}{\partial x_{j}}=F_{j} \frac{\partial u_{i}}{\partial x_{j}}+\lambda \nabla^{2} F_{i}, & \operatorname{div} \mathbf{F}=0, \\
\frac{\partial G_{i}}{\partial t}+u_{j} \frac{\partial G_{i}}{\partial x_{j}}=-G_{j} \frac{\partial u_{j}}{\partial x_{i}}+\lambda \nabla^{2} G_{i}, & \operatorname{curl} \mathbf{G}=0,
\end{array}
$$

where again the velocity $\mathbf{u}$ is independent of $\mathbf{F}$ and $\mathbf{G}$.

In the absence of diffusion, $\mathbf{F}$ is proportional to a material line element and the flux of $\mathbf{F}$ across a material surface is constant; the vector $\mathbf{G}$ can be written as $\nabla \theta$ and is proportional to the vector representing a material surface element. The most important examples of vector fields like $\mathbf{F}$ are the magnetic field $\mathbf{H}$ (with $\lambda=$ magnetic diffusivity) and the vorticity $\omega$ which satisfies (1.2) with $\lambda=\nu$; the magnetic field will be approximately dynamically passive if the magnetic energy density is sufficiently small. Batchelor (1952) has discussed the properties of such vector fields in terms of the effect of homogeneous turbulence on material lines and surfaces and shown that if $\lambda=0$ the mean square values $\overline{\mathbf{F}^{2}}$ and $\overline{\mathbf{G}^{2}}$ grow exponentially with time when the influence of the particular initial distribution has been lost. The amplification exponent in each case is of order $(\epsilon / \nu)^{\frac{1}{2}}$, where $\epsilon$ is the mean rate of kinetic energy dissipation per unit mass, representing the result that the amplification is due primarily to the rate of straining of the fluid that is associated with the small eddies and the vorticity.

Batchelor (1952) considered further the effect of diffusion. For the case $\lambda \ll \nu$, he showed that the amplification of $\mathbf{G}$ is accompanied by a continual decrease of scale and an associated increase in molecular dissipation until a steady state is reached when the length scale $\stackrel{\vec{l}}{G}_{G}$, say, of the G-field is of order $(\lambda / v)^{\frac{1}{2}} l_{d}$, where 
$l_{d}=\left(\nu^{3} / \epsilon\right)^{\frac{1}{4}}$. For the $\mathbf{F}$-field, the argument was put forward that the length scale for dissipation ${ }^{*}$, remains of order $l_{d}$ and that $\overline{\mathbf{F}^{2}}$ grows exponentially without limit in the absence of any back reaction on the velocity distribution (see also Batchelor \& Townsend 1956).

It has become apparent from the work of Pearson (1959) that the argument for the F-field is fallacious. Pearson examined the solution of (1.2) with F initially a stationary random function of position and the velocity field a uniform irrotational distortion or pure strain, $\mathbf{u}=\left(\alpha_{1} x_{1}, \alpha_{2} x_{2}, \alpha_{3} x_{3}\right)$, and it became clear from this work that $\stackrel{*}{l}_{F}$ continually decreases (if initially larger) until it reaches a value of order $\left(-\lambda / \alpha_{3}\right)^{\frac{1}{2}}$ (for definiteness, we shall always suppose that $0<\alpha_{1} \geqslant \alpha_{2} \geqslant \alpha_{3}<0$ ) and in fact it behaves like ${ }_{G}^{*}$. Physically, the fallacy lay in neglecting the decrease in scale in the direction perpendicular to a material line element as it is stretched. Nevertheless, Pearson found that $\overline{\mathbf{F}^{2}}$ continues to grow exponentially with time; this phenomenon will be called the Pearson divergence. The behaviour of $\overline{\mathbf{F}^{2}}$ in a field of homogeneous turbulence is therefore uncertain. The original argument based on the assumption that $l_{F}^{*} \sim l_{d}$ when $\lambda \ll \nu$ is certainly fallacious, and the fact that $\vec{l}_{F}$ seems to behave like ${ }_{l_{*}}^{*}$ would suggest that $\overline{\mathbf{F}^{2}}$ behaves like $\overline{\mathbf{G}^{2}}$ and that the amplification is limited by diffusion effects. On the other hand, in any small region of the fluid it is a plausible hypothesis that the local straining motion is approximately a pure strain so that the Pearson divergence suggests that $\overline{\mathbf{F}^{2}} \rightarrow \infty$ as originally predicted.

The aim of the present paper is to show that Batchelor's (1959) ideas on the small-scale structure favour the first alternative that the growth of $\overline{\mathbf{F}^{2}}$ is limited by diffusion. The Pearson divergence is examined and it is shown to depend on the uniform straining motion being of infinite extent or, alternatively, on the amplification of Fourier components of arbitrarily small wave-number. Since in a field of turbulence, the regions of approximately uniform strain are of finite extent, in fact of linear dimensions of order $l_{d}$, the Pearson divergence is not though to be relevant to the behaviour of the $\mathbf{F}$-field in a field of homogeneous turbulence.

The analysis is applied in $\S 6$ to the particular case of the magnetic field in a good conductor, for the case when the field is sufficiently weak for the motion to be unaffected by Lorentz forces, and the factor by which a weak applied field of length-scale $L$ is amplified is estimated.

The vorticity $\boldsymbol{\omega}=$ curl $\mathbf{u}$ also satisfies equation (1.2) with $\lambda=v$, but is not a dynamically passive quantity. However, the consequences of assuming that the components of the vorticity distribution of scale less than $l_{d}$ satisfy an equation similar to that satisfied by the F-field seem worth examining, and this is done in an appendix. The argument leads to a form of the longitudinal energy spectrum function for very large wave-numbers roughly similar to one given by Townsend (1951 a). 


\section{The equation for the covariance. of $\theta$ for small separation}

We suppose (as is appropriate for the small-scale structure) that the distribution of $\theta$ is statistically isotropic (with $\vec{\theta}=0$ for convenience). The covariance of $\theta$ is $S(r)=\overline{\theta(\mathbf{x}) \theta(\mathbf{x}+\mathbf{r})}=\overline{\theta \theta^{\prime}}$ with an obvious notation. From the observation that the velocity gradients over regions of linear dimensions $l_{d}$ are approximately constant, and the assumptions that the principal axes of rate of strain rotate with the fluid and that level surfaces of $\theta$ are approximately aligned normal to the direction of least rate of strain (or maximum compression), Batchelor (1959) deduced the equation

$$
\frac{\partial S}{\partial t}=-\gamma r \frac{\partial S}{\partial r}+2 \lambda\left(\frac{\partial^{2} S}{\partial r^{2}}+\frac{2}{r} \frac{\partial S}{\partial r}\right) \quad \text { for } \quad r<l_{d}
$$

where $\gamma(<0)$ is an average value of the least principal rate of strain. The evidence for the assumption that the principal axes of rate of strain rotate with the fluid does not seem to be altogether conclusive. It seems to depend mainly on some experiments by Townsend $(1951 b)$ on the cooling of heat spots in grid generated turbulence. However, it can be shown (Alexandrou 1963) that rotation of the principal axes of rate of strain relative to the fluid need not affect significantly the rate of cooling, and that the experimental measurements are consistent with a certain amount of relative rotation. It therefore seems worthwhile to repeat here Batchelor's argument in a form independent of this particular assumption; especially since Alexandrou (1963) has shown that the Pearson divergence in a uniform straining motion does not arise if there is sufficient relative rotation, and moreover that such a relative rotation can leave the rate of heat-spot cooling approximately the same.

It follows from (1.1) in the usual way that

$$
\frac{\partial S}{\partial t}-2 \lambda\left(\frac{\partial^{2} S}{\partial r^{2}}+\frac{2}{r} \frac{\partial S}{\partial r}\right)=\overline{\left(u_{i}-u_{i}^{\prime}\right) \frac{\partial \theta \bar{\theta}^{\prime}}{\partial r_{i}}}=T, \quad \text { say }
$$

The current picture of the properties of homogeneous turbulence based on the work of the last 25 years is that the net effect of the triple interaction or transfer term $T$ in a fully developed situation, when the influence of particular initial conditions has been lost, is to reduce continually the length-scale of the $\theta$-variations. (This is the interpretation in physical space of the well-known cascade process.) The Batchelor approximation

$$
T=-\gamma r \partial S / \partial r
$$

has this property, as is clearly shown by the solution of (2.1) when $\lambda=0$, namely, $S(r)=S_{0}\left(r e^{-\gamma t}\right)$, where $S_{0}$ denotes the initial value at $t=0$.

To derive (2.3), we write

$$
u_{i}^{\prime}-u_{i} \doteqdot \alpha_{i j} r_{j}+(\boldsymbol{\Omega} \wedge \mathbf{r})_{i}
$$

where $\boldsymbol{\Omega}$ is the angular velocity (in space) of the principal axes of rate of strain at the point $\mathbf{x}$ and $\alpha_{i j}$ is the velocity gradient tensor of the motion relative to the principal axes. The rate of strain tensor is $\frac{1}{2}\left(\alpha_{i j}+\alpha_{j i}\right)$. The tensor $\alpha_{i j}$ is not 
in general symmetric, and the asymmetric part describes the rotation of the principal axes relative to the fluid. We now assume, following Batchelor, that the $\alpha_{i j}$ vary little in the time $(\nu / \epsilon)^{\frac{1}{2}}$ (which is the time characteristic of the straining motion), so that the straining motion may be regarded as persistent. $\dagger$ For $\lambda \ll \nu$, we assume that the level surfaces of $\theta$ in the vicinity of $\mathbf{x}$ are orientated like material surfaces and are approximately plane with common normal $\mathbf{n}$, so that in any realization $\theta \theta^{\prime}=\Theta *\left(n_{i} r_{i}\right)$. Then

$$
\alpha_{i j} r_{j} \frac{\partial \theta \theta^{\prime}}{\partial r_{i}}=\alpha_{i j} r_{j} n_{i} \dot{\Theta}^{*}=\gamma r \frac{\partial \theta \theta^{\prime}}{\partial r}
$$

where $\dot{\Theta}^{*}$ denotes the derivative of $\Theta *$ with respect to its argument, if

$$
\gamma n_{j}=\alpha_{i j} n_{i}
$$

Since (2.3) follows from (2.5) on taking averages, assuming that the rigid body rotation with angular velocity $\boldsymbol{\Omega}$ is not correlated statistically with $\partial\left(\theta \theta^{\prime}\right) / \partial r_{i}$, and supposing that fluctuations in $\gamma$ are negligible fractions of the mean value, we see that a sufficient condition for the Batchelor approximation is that in each realization (2.6) is satisfied, i.e. $\mathbf{n}$ is an eigenvector and $\gamma$ an eigenvalue of the matrix $\alpha_{j i}$. Now by hypothesis, $\mathbf{n}$ behaves like a material surface element, and hence

$$
\frac{d n_{i}}{d t}=-\alpha_{j i} n_{j}
$$

where the components $n_{i}$ are taken relative to axes rotating with the angular velocity $\Omega$. Let $\gamma(<0)$ denote the smallest root of the cubic in $s$,

$$
\operatorname{det}\left(\alpha_{i j}-s \delta_{i j}\right)=0 \text {. }
$$

Since $\alpha_{i i}=0$ (condition of incompressibility), the sum of the roots is zero. Then the general solution of (2.7) behaves asymptotically like $\mathbf{n}^{(\gamma)} e^{-\gamma t}$, where the components of $\mathbf{n}^{(\gamma)}$ satisfy $(2,6)$, provided the $\alpha_{i j}$ are approximately constant. Thus if the strain following the motion is approximately persistent, the material surfaces in the vicinity of a point will be aligned with their normals in a direction $\mathrm{n}$ which satisfies (2.6), and (2.3) then follows.

If $\alpha_{i j}$ is a symmetric tensor so that the principal axes of rate of strain do not rotate relative to the fluid, this argument reduces to Batchelor's with $\gamma=\alpha_{3}$, the least principal rate of strain.

The argument fails in this form if (2.8) does not have a negative real root. There are then one positive real root and two complex roots with equal negative real parts. Material surfaces are then not distorted locally into planes, but into portions of elliptical cylinders for the compression now takes place equally in all directions perpendicular to an axis of extension. But we can now write

where

$$
\theta \theta^{\prime}=\Theta *\left(a_{i j} r_{i} r_{j}\right)
$$

$$
\frac{d a_{i j}}{d t}=-\alpha_{k j} a_{k i}-\alpha_{k i} a_{k j}
$$

if again it is assumed that the level surfaces behave like material surfaces. It can be shown by some heavy algebra that the asymptotic solutions of $(2.10)$ are in general proportional to $e^{-\gamma t}$, where $\gamma$ is twice the negative real part of the

$\uparrow$ The evidence for this cited by Batchelor (1959) still seems to apply when the principal axes of rate of strain rotate relative to the fluid. 
complex eigenvalues, and moreover that the $a_{i j}$ are then such that the relation between the first and third terms in (2.5) still holds. Thus the approximate expression for the transfer term holds however rapidly the principal axes of rate of strain rotate relative to the fluid, provided it is still assumed that the level surfaces of $\theta$ are aligned like material surfaces and the local straining motion following the fluid persists sufficiently long for material surfaces to take up asymptotic orientations.

The above argument gives sufficient conditions for (2.3) to hold, but it is far from certain that they are altogether necessary. Thus, we have discussed the requirement that (2.5) holds in each realization, but the necessary condition is that the ensemble averages should be equal; there is no need for the first and third terms to be equal in each realization. The present writer believes that (2.3) is in fact a consequence of dimensional analysis and the assumption that the transfer term decreases the scale. For $r<l_{d}, T$ is linear in the components of the velocity gradient tensor and in the first derivatives of $\theta \theta^{\prime}$. If we ask that its ensemble average have the same properties, and moreover be isotropic and independent of any particular initial conditions (as is appropriate for the quasisteady small-scale structure), then there seems to be no other possibility except (2.3), where $\gamma$ is some average value of the velocity gradients and is of order $\left(\overline{\omega^{2}}\right)^{\frac{1}{2}}$. The condition $\gamma<0$ follows from the requirement that the transfer term decreases the scale, as shown by the solution of (2.1) with $\lambda=0$. (A feature of this argument is that it appears to apply even when $\lambda / \nu$ is not very small, although $\gamma$ depends in some way on the degree of orientation of the level surfaces, i.e. the difference in any realization between a level surface of $\theta$ and a material surface, and since this will be affected by the Prandtl number $\nu / \lambda$ it may be expected that $\gamma$ would be a function of the Prandtl number. If $(2.3)$ is valid for $\lambda \approx \nu$, the high order derivatives of $S$ at $r=0$ can be evaluated, and the high-order moments of the spectrum function of $\theta$ then follow and information about the spectrum function at very large wave-numbers is obtained.)

\section{Behaviour in a uniform straining motion}

Because of the Pearson divergence, it is part of our purpose to examine the extent to which a uniform straining motion of infinite extent reproduces the small-scale features of turbulent motion. We shall now discuss the way in which (2.1) can be derived from the exact solutions for this case. For simplicity and because it was the case examined by Pearson, we shall consider only the case of a pure straining motion $\mathbf{u}=\left(\alpha_{1} x_{1}, \alpha_{2} x_{2}, \alpha_{3} x_{3}\right)$. Then $\overline{\theta \theta^{\prime}}=\Theta(\mathbf{r}, t)$ satisfies

$$
\frac{\partial \Theta}{\partial t}+\alpha_{1} r_{1} \frac{\partial \Theta}{\partial r_{1}}+\alpha_{2} r_{2} \frac{\partial \Theta}{\partial r_{2}}+\alpha_{3} r_{3} \frac{\partial \Theta}{\partial r_{3}}=2 \lambda \nabla^{2} \Theta \text {. }
$$

The general solution of this equation is (as may be verified by substitution)

where

$$
\Theta(\mathbf{r}, t)=\frac{1}{(2 \pi)^{\frac{3}{2}} a_{1} a_{2} a_{3}} \int \exp \left[-\sum_{\mu=1}^{3} \frac{\left(r_{\mu}-r_{\mu 0} e^{\alpha_{\mu} l}\right)^{2}}{2 a_{\mu}^{2}}\right] \Theta\left(\mathbf{r}_{0}, 0\right) d \mathbf{r}_{0}, \dagger
$$

$\dagger$ The summation convention does not apply to the suffix $\mu$. 
For definiteness, we shall suppose $\alpha_{2}>0$. (This in fact seems to be the more appropriate case since $\overline{\alpha_{1} \alpha_{2} \alpha_{3}}<0$ for homogeneous turbulence when the $\alpha_{\mu}$ denote the principal rates of strain.) The quantity $\Theta$ is clearly highly anisotropic, but we can obtain an isotropic quantity $\tilde{S}(r, t)$ by averaging over all orientations of the axes and taking the initial distribution as a function of $r_{0}$ alone. The averaging is equivalent to taking the mean value of $\Theta$ over the sphere $|\mathbf{r}|=r$.

In order to bring out the main features of the problem with a minimum of analysis, we restrict ourselves here to a simple example

$$
\Theta\left(\mathbf{r}_{0}, 0\right)=\exp \left[-\frac{1}{2} r_{0}^{2} / l^{2}\right]
$$

The results obtained for large $t$ are quite typical. The solution of (2.11) is then

$$
\Theta(\mathbf{r}, t)=\frac{l^{3}}{a_{1}^{\prime} a_{2}^{\prime} a_{3}^{\prime}} \exp \left[-\frac{1}{2}\left(\frac{r_{1}^{2}}{a_{1}^{\prime 2}}+\frac{r_{2}^{2}}{a_{2}^{\prime 2}}+\frac{r_{3}^{2}}{a_{3}^{\prime 2}}\right)\right]
$$

where

$$
a_{\mu}^{\prime}=a_{\mu}\left(1+l^{2} e^{2 \alpha_{\mu} t} / a_{\mu}^{2}\right)^{\frac{1}{2}}
$$

In our case, $a_{1}^{\prime}$ and $a_{2}^{\prime}$ tend to infinity as $t \rightarrow \infty$, and $a_{3}^{\prime} \rightarrow\left(-2 \lambda / \alpha_{3}\right)^{\frac{1}{2}}$. Let us now examine the equation satisfied by

$$
\widetilde{S}(r, t)=\frac{1}{4 \pi r^{2}} \int_{|\mathbf{r}|=r} \Theta(\mathbf{r}, t) d A
$$

It follows from (2.11) and (2.15) that in this case $\widetilde{S}$ satisfies the equation

$$
\frac{\partial \widetilde{S}}{\partial t}+K r \frac{\partial \widetilde{S}}{\partial r}=2 \lambda\left(\frac{\partial^{2} \widetilde{S}}{\partial r^{2}}+\frac{2}{r} \frac{\partial \widetilde{S}}{\partial r}\right)
$$

where $\quad K=\int_{|\mathrm{r}|=r}\left(\sum_{\mu=1}^{3} \frac{\alpha_{\mu} r_{\mu}^{2}}{a_{\mu}^{\prime 2}}\right) \Theta d A / \int_{|\mathrm{r}|=r}\left(\sum_{\mu=1}^{3} \frac{r_{\mu}^{2}}{a_{\mu}^{\prime 2}}\right) \Theta d A$

It now follows that if $r \ll a_{2}^{\prime} \sim\left(2 \lambda / \alpha_{2}\right)^{\frac{1}{2}} e^{\alpha_{2} t}$ for large $t$, then

and if $r>a_{1}^{\prime} \sim\left(2 \lambda / \alpha_{1}\right)^{\frac{1}{2}} e^{\alpha_{1} t}$, then

$$
K \approx \alpha_{3}<0
$$

$$
K \approx \alpha_{1}>0 \text {. }
$$

Thus the average of a uniform straining motion gives a behaviour for small $r$ in agreement with (2.1), but for large $r$ it gives a different behaviour and one, moreover, which conflicts with the idea that the transfer term reduces the scale; for $T=-\alpha_{1} r \partial S / \partial r$ would increase the scale of the fluctuations. Physically, this is due to the fact that a blob of $\theta$ in this infinite uniform straining motion is pulled out into a thin plane disk of ever increasing area so that there is always an increase in the scale of the variations at some separation. A similar result holds for the case $\alpha_{2}<0$.

Such a difference at large separations is of course to be expected. The question is (and in view of the Pearson divergence it is particularly relevant): does this difference affect the properties at small separations? The answer seems to be yes. For consider the solution of (2.1) with the initial distribution (2.14) and let us suppose that $l<l_{d}$ so that the initial distribution lies within the region of 
uniform strain. It can be shown (and may be verified by substitution) that the solution is

$$
S(r, t)=\frac{l^{3}}{a^{\prime 3}} e^{3 \gamma t} e^{-\frac{1}{2} r^{2} / a^{\prime 2}} ; \quad a^{\prime 2}=\frac{2 \lambda}{-\gamma}+\left(l^{2}+\frac{2 \lambda}{\gamma}\right) e^{2 \gamma t}
$$

In particular,

$$
\overline{\theta^{2}}=S(0, t) \propto e^{3 \gamma t} .
$$

On the other hand, the behaviour of $\theta$ in a field of uniform strain gives from (2.15) (which is in fact valid if $\alpha_{2}$ is positive or negative)

$$
\tilde{S}(0, t)=\Theta(0, t)=\frac{l^{3}}{a_{1}^{\prime} a_{2}^{\prime} a_{3}^{\prime}} \propto\left\{\begin{array}{lll}
e^{\alpha_{3} t} & \text { if } & \alpha_{2}>0, \\
e^{-\alpha_{1} t} & \text { if } & \alpha_{2}<0 .
\end{array}\right\}
$$

For comparison, we should put $\gamma=\alpha_{3}$ in (2.23), for this would be the value of $\gamma$ if the principal axes of rate of strain did not rotate relative to the fluid in which case $\gamma$ is equal to an average value of the least principal rate of strain (Batchelor 1959). The qualitative behaviour of (2.23) and (2.24) is the same, but the different exponent of decay turns out to be all important when the analysis is applied to the $\mathbf{F}$-field. Since $\alpha_{1}+\alpha_{2}+\alpha_{3}=0, \frac{1}{2}<-\alpha_{3} / \alpha_{1}<2$, and therefore $\overline{\theta^{2}}$ as given by (2.23) decays more rapidly than $\bar{\theta}^{2}$ in a uniform straining motion of infinite extent. The less rapid dissipation by the infinite straining field is due to the increase in scale that goes on at sufficiently large $r$ and which enables the spatial variation of $\theta$ to survive longer against molecular diffusion. Mathematically, the difference is due to the different shapes at large $r$ of the characteristic curves in the $(r, t)$-plane of equations (2.1) and (2.18) with $\lambda=0$. For (2.1), the characteristics are always pointed towards the $r$-axis, whereas for (2.18) there are always characteristics at large values of $r$ pointed away from the $r$-axis. The asymptotic behaviour near $r=0$ for large $t$ is dominated by these characteristics which eventually bend round and approach the $r$-axis. In other words, for the uniform straining field the behaviour for small $r$ is always affected by the conditions for arbitrarily large values of $r$. The interpretation in wave-number space of this phenomenon will be considered in $\S 4$.

If, however, we suppose that a steady distribution of $\theta$-variations is maintained by a continuous source of fluctuations of length-scale $l$, the steady values of $S$ and $\widetilde{S}$ can be obtained by integrating the unsteady solutions $S(r, t)$ and $\tilde{S}(r, t)$ for an instantaneous source with respect to $t$ from 0 to $\infty$. Since according to (2.18) and (2.20), $S$ and $\widetilde{S}$ satisfy the same equation for $r<l$, it is clear that the steady solutions will have the same dependence upon $r$ for values of $r<l$ but very different behaviour for larger values of $r$. Because of the different rates of decay of the unsteady solutions, the source strength to maintain a given level of $\theta$-variations has to be $3 \gamma / \alpha_{3}$ or $-3 \gamma / \alpha_{1}$ (according as $\alpha_{2}>$ or $<0$ ) times greater if the Batchelor approximation holds than if the $\theta$-variations behave as if in a uniform infinite straining field. This difference may seem slight or unimportant, but as we shall see in the next section, where the present ideas are applied to the $\mathbf{F}$ and $\mathbf{G}$-fields, it is at the heart of the Pearson divergence. 


\section{The equation for the covariance tensors of $F$ and $G$ for small separation}

We assume statistically isotropic distributions. Then the covariance tensors have the form (see Batchelor 1953)

and

$$
\begin{gathered}
\overline{F_{i} F_{j}^{\prime}}=\left(f+\frac{1}{2} r \frac{\partial f}{\partial r}\right) \delta_{i j}-\frac{1}{2 r} \frac{\partial f}{\partial r} r_{i} r_{j}, \\
\overline{G_{i} G_{j}^{\prime}}=g \delta_{i j}+\frac{1}{r} \frac{\partial g}{\partial r} r_{i} r_{j},
\end{gathered}
$$

where $f$ and $g$ are scalar functions of the separation magnitude $r$. The contracted tensors are

and

$$
\overline{F_{i} \bar{F}_{i}^{\prime}}=3 f+r \frac{\partial f}{\partial r}=F(r)
$$

Note that $\overline{\mathbf{F}^{2}}=F(0), \overline{\mathbf{G}}^{\overline{2}}=G(0)$. In the usual way, we obtain from (1.2) and (1.3),

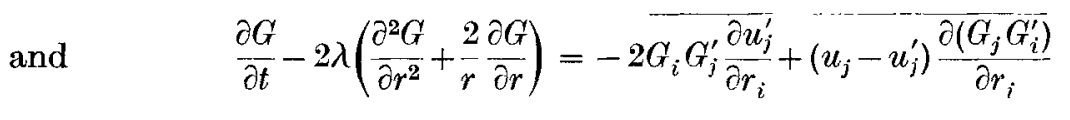

$$
\begin{aligned}
\frac{\partial F}{\partial t}-2 \lambda\left(\frac{\partial^{2} F}{\partial r^{2}}+\frac{2}{r} \frac{\partial F}{\partial r}\right) & =2 F_{i} F_{j}^{\prime} \frac{\partial u_{i}^{\prime}}{\partial r_{j}}+\overline{\left(u_{j}-u_{j}^{\prime}\right) \frac{\partial\left(F_{i} F_{i}^{\prime}\right)}{\partial r_{j}}} \\
& =T_{F}, \text { say; }
\end{aligned}
$$

$$
=T_{G}, \text { say. }
$$

We now consider the form taken by the transfer terms $T_{F}$ and $T_{Q}$ for small values of $r$ on the basis of the assumptions used for the $\theta$-covariance in the previous section. We use (2.5) and take axes rotating with the principal axes of rate of strain, and again assume that the $\alpha_{i j}$ vary slowly with time. Then relative to these axes, that are also moving with the fluid,

$$
\frac{\partial F_{k}}{\partial t}+\alpha_{i j} x_{j} \frac{\partial F_{k}}{\partial x_{i}}=\alpha_{k j} F_{j}+\lambda \nabla^{2} F_{k^{*}}
$$

This equation has the general solution

$$
\mathbf{F}=\sum_{p=1}^{3} \mathbf{1}^{(p)}(t) \theta^{(p)}(\mathbf{x}, t)
$$

where the summation convention does not apply to the superfix $p$, and

$$
\frac{d l_{i}^{(p)}}{d t}=\alpha_{i j} l_{j}^{(p)} ; \quad \frac{\partial \theta^{(p)}}{\partial t}+\alpha_{i j} x_{j} \frac{\partial \theta^{(p)}}{\partial x_{i}}=\lambda \nabla^{2} \theta^{(p)} .
$$

Thus the vectors $\mathbf{1}^{(p)}$ behave like material line elements and the quantities $\theta^{(p)}$ like conserved scalars. Assuming again that the local strain is persistent so that line elements and material surfaces take up an asymptotic orientation, we have that

$$
\mathbf{F}=\mathbf{1}(t) \theta(\mathbf{n} \cdot \mathbf{x})
$$


where 1 is proportional to $e^{\alpha t}, \alpha$ being the greatest root of (2.8), and

and $\mathbf{n}$ satisfies (2.6).

$$
\alpha l_{i}=\alpha_{i k} l_{k},
$$

Similarly, we find

$$
\mathbf{G}=\mathbf{n} \theta(\mathbf{n} \cdot \mathbf{x}) .
$$

The vectors 1 and $\mathbf{n}$ are clearly perpendicular in accordance with the assumption that $\mathbf{F}$ is aligned asymptotically along material lines and $\mathbf{G}$ normal to material surfaces (see Batchelor 1952).

Substituting (3.10) into (3.5), we obtain (again writing $\theta \theta^{\prime}=\Theta^{*}(\mathbf{n} . \mathbf{r})$ )

$$
\begin{aligned}
T_{F} & =\overline{2 \alpha_{i j} l_{i} l_{j} \theta \theta^{\prime}}-\overline{\alpha_{j k} r_{k} l_{i}^{2} \frac{\overline{\partial \Theta}{ }^{*}}{\partial r_{j}}} \\
& =\overline{2 \alpha l_{i}^{2} \theta \theta^{\prime}}-\overline{\gamma r} \overline{\frac{\partial}{\partial r}\left(l_{i}^{2} \theta \theta^{\prime}\right)} \text { by (3.11) and (2.5) } \\
& =2 \alpha F-\gamma r \frac{\partial F}{\partial r}
\end{aligned}
$$

where it has again been assumed in the last step that fluctuations in $\alpha$ and $\gamma$ are negligible fractions of the mean values. Similarly, we obtain on substituting (3.12) into (3.6)

$$
T_{C}=-2 \gamma G-\gamma r \frac{\partial G}{\partial r} \text {. }
$$

These are the expressions for the transfer functions analogous to (2.3) for the transfer function of $\theta$. The argument given here assumes that the eigenvalues of $\alpha_{i j}$ are real. If this is not so, then the case in which the complex roots have negative real parts will go through as before (see (2.9) and (2.10)) since $\alpha$ is still real. If the complex roots have positive real part $\alpha_{r}$, then the step in (3.13) when we write $l_{i} l_{j} \alpha_{i j}=\alpha l_{i}^{2}$ still holds with $\alpha=\alpha_{r}, \gamma$ now being real and negative.

The expression (3.14) can of course be derived from (2.1) and the exact relation $G=-\nabla^{2} S$, and is implicit in Batchelor's (1959) work, provided $G$ is the gradient of a stationary random function of position. The separate derivation is, however, useful because the scalar quantity of which $\mathbf{G}$ is necessarily the gradient (since $\operatorname{curl} \mathbf{G}=0$ ) need not be a stationary random function.

According to the present derivation, $\alpha(>0)$ is the exponent in the exponential law for the increase in length of line elements, and $\gamma(<0)$ that in the expression for the decrease of normal distance between material surfaces. If the principal axes of rate of strain do not rotate too rapidly relative to the fluid (although as mentioned earlier the evidence for this is not conclusive) we will have $\alpha \sim \overline{\alpha_{1}}$, and $\gamma \sim \overline{\alpha_{3}}$.

The physical significance of the two terms in the expressions for $T_{F}$ and $T_{G}$ is clear. The first term describes the amplification of $\mathbf{F}$ due to the stretching of material lines (Batchelor 1952), and the second term the decrease in scale due to the convection. For $\mathbf{G}$, the process which decreases the scale also causes the amplification which explains why $\gamma$ occurs in both terms. 
Thus, an application of the ideas introduced by Batchelor leads to the hypothesis that, for $r<l_{d}$, the scalar quantities $F$ and $G$ satisfy the equation

$$
\frac{\partial H}{\partial t}=2 A\left(H+\frac{r}{2 \sigma} \frac{\partial H}{\partial r}\right)+2 \lambda\left(\frac{\partial^{2} H}{\partial r^{2}}+\frac{2}{r} \frac{\partial H}{\partial r}\right),
$$

where for the F-field, $A=\alpha$ and $\sigma=-\alpha / \gamma$; and for the G-field, $A=-\gamma$ and $\sigma=1$. Moreover, the argument given previously for the $\theta$-covariance that the approximate form for small $r$ is a consequence of dimensional arguments and the structure of the exact form of the transfer terms will apply to $T_{F}$ and $T_{G}$ with equal validity. There is therefore reason to believe, and at the least it is a reasonable working hypothesis, that (3.15) holds even if the orientation is not complete or the strain not sufficiently persistent (and that it may be applicable when $\lambda \approx \nu$, with the proviso that $A$ and $\sigma$ may depend upon the Prandtl number). As regards the value of $\sigma$ for the $\mathbf{F}$-field, the fact that $\overline{\alpha_{1} \alpha_{2} \alpha_{3}}<0$ suggests that $\alpha<-\gamma$ and therefore $\sigma<1$. Batchelor \& Townsend (1956) give a value of 0.78 for the ratio $-\overline{\alpha_{1}} \sqrt{\alpha_{3}}$.

Equation (3.15) enables us to discuss the fine-scale structure of the vector fields and the question whether dissipation can hold the amplification in check. But before doing this, we must consider because of the Pearson divergence the behaviour of the fields in an infinite uniform strain.

\section{Behaviour in a uniform straining motion}

Again for the sake of simplicity we take the case of a pure strain and examine the behaviour of stationary random distributions of $\mathbf{F}$ and $\mathbf{G}$. It is immediate that the components $F_{\mu}$ and $G_{\mu}$ can be written

$$
F_{\mu}=e^{\alpha_{\mu} t} \theta_{F}^{(\mu)} ; \quad G_{\mu}=e^{-\alpha_{\mu} t} \theta_{G}^{(\mu)},
$$

where each $\theta$ satisfies the scalar diffusion equation. Hence with an obvious notation

$$
\overline{F_{1} F_{1}^{\prime}}=e^{2 \alpha_{1} t} \Theta_{F^{\prime}}^{(1)}(\mathbf{r}, t) ; \quad \overline{G_{1} G_{1}^{\prime}}=e^{-2 \alpha_{1} t} \Theta_{G}^{(1)}(\mathbf{r}, t) ; \quad \text { etc. }
$$

where the $\Theta$ are given by (2.12) in terms of initial values. These quantities are not isotropic, but isotropic quantities can be obtained by averaging over all orientations of the axes or equivalently by integrating over the surface of a sphere as deseribed by (2.17). It then follows as in $\S 2$ that the average of $\overline{F_{i}} \bar{F}_{i}^{\prime}$ constructed in this way is asymptotically proportional to $e^{2 \alpha_{1} t} \tilde{S}_{F}$, where $\widetilde{S}_{F}$ satisfies (2.18), and similarly the average of $\overline{G_{i} G_{i}^{\prime}}$ is proportional to $e^{-2 \alpha_{3} t} \widetilde{S}_{G}$, where $\widetilde{S}_{G}$ also satisfies (2.18). Denoting these averages by $\tilde{H}$, we have that

$$
\frac{\partial \tilde{H}}{\partial t}=2 A \tilde{H}-K r \frac{\partial \tilde{H}}{\partial r}+2 \lambda\left(\frac{\partial^{2} \tilde{H}}{\partial r^{2}}+\frac{2}{r} \frac{\partial \tilde{H}}{\partial r}\right),
$$

where $A=\alpha_{1}$, or $A=-\alpha_{3}$, and $K$ has the properties described by (2.20) and (2.21); i.e. it depends upon the initial distribution and the time $t$ but has the property that for small $r, K \approx \alpha_{3}$, and for large $r, K \approx \alpha_{1}$. This equation is to be compared with (3.15). 
Now although these equations have the same form over an increasing region of $r$ including $r=0$, their solutions near $r=0$ differ significantly in the way they depend upon the time. Consider for definiteness an initial distribution

$$
H\left(r_{0}, 0\right)=\left(1-\frac{r_{0}^{2}}{3 l^{2}}\right) \exp \left[-\frac{1}{2} r_{0}^{2} / l^{2}\right]
$$

since by virtue of (3.3) and (3.4), $F$ and $G$ must both satisfy

$$
\int_{0}^{\infty} r^{2} F d r=\int_{0}^{\infty} r^{2} G d r=0
$$

For the behaviour in a uniform straining motion, the corresponding initial conditions will be

$$
\Theta_{F}^{(1)}(\mathbf{r}, 0)=\frac{1}{3}\left(1-\frac{r_{2}^{2}+r_{3}^{2}}{2 l^{2}}\right) e^{-\frac{1}{2} r^{2} / l^{2}} ; \quad \Theta_{\pi}^{(3)}(\mathbf{r}, 0)=\frac{1}{3}\left(1-\frac{r_{3}^{2}}{l^{2}}\right) e^{-\frac{1}{2} r^{2} / l^{2}} ; \quad \text { etc. }
$$

It follows, by the use of (2.12), that the infinite uniform straining motion gives

$$
\begin{aligned}
& \overline{F_{1}^{2}}=\frac{l^{3}}{3 a_{1}^{\prime} a_{2}^{\prime} a_{3}^{\prime}}\left\{1-\frac{1}{2} \frac{a_{2}^{2}}{a_{2}^{\prime 2}}-\frac{1}{2} \frac{a_{3}^{2}}{a_{3}^{\prime 2}}\right\} e^{2 \alpha_{1} t} \\
& \overline{G_{3}^{2}}=\frac{l^{3}}{3 a_{1}^{\prime} \overline{a_{2}^{\prime}}}\left\{1-\frac{a_{3}^{2}}{a_{3}^{\prime 2}}\right\} e^{-2 \alpha_{3} t} ; \text { etc. }
\end{aligned}
$$

where $a_{1}$ and $a_{1}^{\prime}$, etc., are given by (2.13) and (2.16). We now find that for large $t$,

$$
\begin{gathered}
\overline{F^{2}} \propto\left\{\begin{array}{lll}
e^{\left(\alpha_{1}-\alpha_{2}\right) t} & \text { if } & \alpha_{2}>0 ; \\
e^{\left(\alpha_{2}-\alpha_{3}\right) t} & \text { if } & \alpha_{2}<0 ;
\end{array}\right\} \\
\overline{G^{2}} \propto\left\{\begin{array}{lll}
e^{\alpha_{3} t} & \text { if } & \alpha_{2}>0 ; \\
e^{-\alpha_{1} t} & \text { if } & \alpha_{2}<0 .
\end{array}\right\}
\end{gathered}
$$

(The results (3.24) are of course included in Pearson's 1959 analysis.)

On the other hand, the solution of (3.15) with the same initial conditions gives

$$
H(r, t)=\frac{l^{5}}{a^{\prime 5}} e^{\left(2-5 \sigma^{-1}\right) A t}\left(1-\frac{r^{2}}{3 a^{\prime 2}}\right) e^{-\frac{1}{2} r^{2} / a^{\prime 2}},
$$

where $a^{\prime}$ is given by (2.22). In particular

$$
F(0) \propto e^{(2 \alpha+5 \gamma) t} ; \quad G(0) \propto e^{3 \gamma t} .
$$

To compare (3.27) with (3.24) and (3.25), we should put $\alpha=\alpha_{1}, \gamma=\alpha_{3}$, for the reason given earlier in connexion with (2.23) and (2.24). The behaviour of $\overline{\mathbf{G}^{2}}$ is the same as that of $\overline{\theta^{2}}$, which is as expected, and the remarks made in $\S 2$ apply here. The difference in the expressions for $\overline{\mathbf{F}^{2}}$ is more important, since according to (3.24) $\overline{\mathbf{F}^{2}} \rightarrow \infty$ (this is the Pearson divergence), whereas from (3.27) $\overline{\mathbf{F}^{2}} \rightarrow 0$ if $\sigma<\frac{5}{2}$ (it will be remembered that $\sigma$ is expected to be less than one). Each result is correct in its own context, and we must again conclude that the application of Batchelor's ideas to the F-and G-fields is not entirely equivalent to considering the behaviour of random distributions in straining motions of infinite extent. What we have to decide is which (if any) result or analysis is appropriate to the fine-scale structure in a field of homogeneous turbulence. 
Now we have seen that the two approaches to the fine-scale structure lead to the same equation being satisfied by $H$ and $\tilde{H}$ for sufficiently small values of $r$, which lends strength to the belief that the equation itself may be realistic. The difference lies in the different time behaviour especially when we consider the existence of steady solutions for the F-field. For according to the uniform strain analysis, the solution for an instantaneous source of $\mathbf{F}$-variations gives $\overline{\mathbf{F}^{2}} \rightarrow \infty$ with time, and therefore a steady solution does not exist. On the other hand, if $\sigma<\frac{5}{2}$ the solution for the covariance function based on the transfer term hypothesis (3.13) has the same dependence on $r$ for small $r$ but tends to zero as $t \rightarrow \infty$. A steady solution can then be obtained by evaluating $\int_{0}^{\infty} F(r, t) d t$, so that $F$ and $G$, and hence $\overline{\mathbf{F}^{2}}$ and $\overline{\mathbf{G}^{2}}$, have the same qualitative behaviour. For the $\mathbf{G}$ - and $\theta$-distributions, the different time behaviour of the two approaches does not matter, for each approach admits of steady solutions being maintained by a continuous source of variations, and these solutions must then necessarily have the same dependence on $r$ for sufficiently small values of $r$ since the same equation is satisfied.

The mathematical reason for the different time behaviour for the $\mathbf{F}$-field is clear. In constructing the solutions of (3.15) and (3.18), it is assumed that these equations are valid for all $r$. The structure of (3.18) is such that the form of the equation for arbitrarily large $r$ determines the asymptotic behaviour of the solution for small $r$ whatever the initial conditions. Hence, the asymptotic behaviour (3.24), which gives the Pearson divergence, depends on the pure straining motion being of infinite extent and is therefore not relevant to a study of the fine-scale structure in homogeneous turbulence where regions of uniform rate of strain are of finite extent. Indeed, a very simple example of the error is provided by the fate of an initially uniform field $\mathbf{F}=$ constant. In homogeneous turbulence, $\overline{\mathbf{F}}$ remains constant, but for a pure straining motion the average of F over all orientations increases asymptotically like $e^{\alpha_{1}{ }^{t}}$. The conclusion seems to be that the Pearson divergence does not prove anything for homogeneous turbulence.

We are left now with equation (3.15) for $r<l_{d}$. Is this equation correct? A point in its favour is that it can be obtained from consideration of the behaviour in an infinite uniform straining motion for $r$ not too large. Also, it is to be emphasized that this equation is not in itself inconsistent with the Pearson divergence. The point is that for all values of $\sigma$ this equation possesses solutions which grow exponentially with time, or are steady, or decay exponentially with time (see $\S 5$ below), and the Pearson divergence arises in effect by feeding in, at large values of $r$, values of $F$ which force an exponentially growing solution. In other words, we can make the solution of (3.15) for $r<l_{d}$ grow, decay, or be steady by requiring that it join on to a growing, decaying, or steady set of values for $r>l_{d}$ where equation (3.15) is not itself valid. The Pearson divergence is not relevant to homogeneous turbulence because the assumption that the uniform straining motion is of infinite extent causes the covariance for sufficiently large $r$ to grow with time which in turn forces the covariance for small $r$ to grow. It is worth pointing out again that the Pearson divergence disappears if the principal 
axes of rate of strain rotate sufficiently rapidly relative to the fluid (Alexandrou 1963), whereas the transfer hypotheses (3.13) and (3.14), which lead to (3.15), are independent of this relative rotation, so that in this case the two approaches would be effectively equivalent. At the very least, it would seem that (3.15) is a reasonable working hypothesis with which to discuss the fine-scale structure.

It may be asked why the arguments for (3.13) and (3.14) lead to an equation which differs for large $r$ from (3.18), for these arguments in the form developed from Batchelor's treatment assume uniform straining. The answer is that the arguments also assumed asymptotic orientation of level surfaces and line elements. This seems permissible in a domain of limited size (in our case of linear dimension less than $l_{d}$ ) but could not be true in a domain of infinite size where the curvature of level surfaces on a large scale may not be neglected. The equivalent assumption in the argument based on dimensions and the exact form of the transfer term is that the transfer term always decreases the scale; the effective transfer term as given by the uniform infinite straining motion increases the scale at large $r$.

Before we discuss the solutions of (3.15) that seem appropriate to homogeneous turbulence, it seems useful to examine the form taken in wave-number space by the present analysis. This will also throw more light on the nature of the Pearson divergence.

\section{The equation for the spectrum functions}

We define the spectrum function of $\theta$ with respect to wave-number magnitude $k$ by the relation

$$
\Gamma(k)=\frac{2}{\pi} \int_{0}^{\infty} k r S(r, t) \sin k r d r .
$$

(For ease of writing, the explicit dependence of the spectrum functions on $t$ will not be shown.) The spectrum functions $\Psi_{F}(k), \Psi_{G}(k), \Psi_{H}(k)$ of $F(r, t)$, $G(r, t), H(r, t)$ are defined similarly. The reverse transforms are

$$
S(r, t)=\int_{0}^{\infty} \Gamma(k) \frac{\sin k r}{k r} d k, \quad \text { etc. }
$$

Note that

$$
S(0, t)=\int_{0}^{\infty} \Gamma(k) d k, \quad H(0, t)=\int_{0}^{\infty} \Psi_{H}(k) d k .
$$

If $S(r, t)$ satisfies $(2.1)$ and $H(r, t)$ satisfies (3.15), together with the conditions that $S(0, t)$ and $H(0, t)$ are finite, it follows on multiplying the equations by $r \sin k r$ and integrating that $\Gamma(k)$ and $\Psi_{H}(k)$ satisfy for $k \ll\left(\epsilon / \nu^{3}\right)^{3}=k_{d}$,

$$
\frac{\partial \Gamma}{\partial t}=\gamma \Gamma+\gamma k \frac{\partial \Gamma}{\partial k}-2 \lambda k^{2} \Gamma
$$

and

$$
\frac{\partial \Psi_{H}}{\partial t}=A\left(2-\sigma^{-1}\right) \Psi_{H}-\frac{A}{\sigma} k \frac{\partial \Psi_{H}}{\partial k}-2 \lambda k^{2} \Psi_{H}
$$

Batchelor (1959), in his analysis of the $\theta$-fluctuations, first worked in wavenumber space and the equation (4.4) is implicit in his work. This was based on the fate of a single Fourier component in a uniform pure straining motion. 
Novikov (1962) has applied Batchelor's treatment in wave-number space without significant change to a weak Fourier component of vorticity and the equation (4.5) (with $\lambda=\nu$ ) is implicit in his work. Novikov's treatment is, however, deficient as wave-number vectors initially nearly parallel to the axis of greatest rate of expansion are neglected entirely and these in fact produce the Pearson divergence for an F-type field like vorticity.

A general solution of (4.4) is

where

$$
\begin{gathered}
\Gamma(k)=e^{\gamma t} \Gamma_{0}(\chi) e^{-\frac{1}{2} b^{2} \chi^{2}}, \\
\chi=k e^{\gamma t}, \\
b^{2}=\frac{2 \lambda}{-\gamma}\left(e^{-2 \gamma t}-1\right),
\end{gathered}
$$

and $\Gamma_{0}(\chi)$ is the value at time $t=0$. According to this solution, a Fourier component of initial wave-number $\chi$ is translated along the wave-number axis, its amplitude being reduced by dissipation. The solution may be expected to be valid if the wave-numbers have magnitude somewhat greater than $k_{d}$. Notice that if $\Gamma_{0}(\chi)$ is zero for $\chi<k_{d}$, then $\Gamma(k)$ is always zero for $k<k_{d}$ and the solution is therefore consistent with the assumptions.

Similarly, a general solution of $(4.5)$ is

$$
\Psi_{H}(k)=e^{(2 A+\gamma) t} \Psi_{0 I I}(\chi) e^{-\frac{1}{2} b^{2} \chi^{2}} .
$$

Consider now the behaviour of a Fourier component of $\theta$ in a pure straining motion. We define a three-dimensional spectrum function

$$
\Delta(\mathbf{k})=\frac{1}{8 \pi^{3}} \int \Theta(\mathbf{r}, t) e^{-\mathbf{i k . r}} d \mathbf{r}
$$

Then

$$
\frac{\partial \Delta}{\partial t}-\alpha_{1} k_{1} \frac{\partial \Delta}{\partial k_{1}}-\alpha_{2} k_{2} \frac{\partial \Delta}{\partial k_{2}}-\alpha_{3} k_{3} \frac{\partial \Delta}{\partial k_{3}}=-2 \lambda k^{2} \Delta
$$

where $k=|\mathbf{k}|$. A general solution of (4.11) is (Pearson 1959)

where

$$
\Delta(\mathbf{k})=\exp \left[-\frac{1}{2}\left(b_{1}^{2} \chi_{1}^{2}+b_{2}^{2} \chi_{2}^{2}+b_{3}^{2} \chi_{3}^{2}\right)\right] \Delta_{0}(\chi)
$$

and

$$
\begin{gathered}
b_{\mu}^{2}=\frac{2 \lambda}{\alpha_{\mu}}\left(1-e^{-2 \alpha_{\mu} t}\right) \\
\chi_{\mu}=k_{\mu} e^{\alpha_{\mu} t},
\end{gathered}
$$

and $\Delta_{0}$ is the value at time $t=0$. The function $\Delta$ is of course non-isotropic, but we can integrate over the surface $|\mathbf{k}|=k$ and obtain

$$
\widetilde{\Gamma}(k)=\int_{|\mathbf{k}|=k} \Delta(\mathbf{k}) d A
$$

where this $\tilde{\Gamma}$ is related to the corresponding $\tilde{S}$ obtained in the same way (see equation (2.17)) by (4.1).

Suppose for definiteness $\alpha_{2}>0$, then for large $t(4.12)$ becomes

$$
\Delta(\mathbf{k})=\exp \left[-\lambda\left(\frac{\chi_{1}^{2}}{\alpha_{1}}+\frac{\chi_{2}^{2}}{\alpha_{2}}-\frac{k_{3}^{2}}{\alpha_{3}}\right)\right] \Delta_{0}(\chi)
$$


We note that provided $\Delta_{0}(\chi)$ is not zero on the $\chi_{1} \chi_{2}$-plane, there are eventually contributions to $\Delta(\mathbf{k})$ from wave-numbers of all magnitudes less than $k_{d}$ even if $\Delta_{0}(\chi)$ is zero for $|\chi|<k_{d}$. Thus in an infinite uniform strain, there is a transfer of spectral density towards zero wave-number and the solution for $\widetilde{\Gamma}(k)$ is very different for small $k$ from that for $\Gamma(k)$ as given by (4.6). Moreover, the spectrum $\widetilde{\Gamma}(k)$ for large $k$ is eventually dominated by wave-number vectors that at some stage transfer spectral density towards $k=0$, and in fact have approached arbitrarily close to $k=0$. To see this, consider the contributions to $\widetilde{\Gamma}(k)$ for a large value of $k>k_{d}$. For sufficiently large $t$, it follows from (4.15) and (4.16) that these are from wave-number vectors with initial components $\chi_{3}=k e^{\alpha_{3}}$ and arbitrary $\chi_{1}$ and $\chi_{2}$ (less than $\left.k_{d} e^{\alpha_{2} t}\right)$. Now at time $t^{\prime}$, the components of this wave-number vector are

$$
\mathbf{k}^{\prime}=\left(\chi_{1} e^{-\alpha_{1} t^{\prime}}, \chi_{2} e^{-\alpha_{2} t^{\prime}}, k e^{\alpha_{3}\left(t-t^{\prime}\right)}\right)
$$

and if $t$ is sufficiently large, there is a wide range of values of $t^{\prime}\left(0<t^{\prime}<t\right)$ for which $\left|\mathbf{k}^{\prime}\right|$ is arbitrarily small. This wave-number vector may initially and finally have magnitude much greater than $k_{d}$, but it spends a long time near the origin of wave-number space and in homogeneous turbulence it is clearly invalid to treat such Fourier components as if in a uniform infinite straining motion.

Nevertheless, as far as the $\theta$-spectrum is concerned no serious inconsistency arises from considering such vectors, since, from (4.15) and (4.16), we have for large $k$ and $t$ that the main contribution to the integral comes from the vicinity of the $k_{3}$-axis; thus

$\tilde{\Gamma}(k) \sim \iint \exp \left[-\lambda\left(\frac{\chi_{1}^{2}}{\alpha_{1}}+\frac{\chi_{2}^{2}}{\alpha_{2}}-\frac{k^{2}}{\alpha_{3}}\right)\right] \Delta_{0}\left(\chi_{1}, \chi_{2}, k e^{\alpha_{3} t}\right) e^{\alpha_{3} l} d \chi_{1} d \chi_{2}=e^{\alpha_{3} l} e^{\lambda k^{2} / \alpha_{3}} \mathfrak{\xi}\left(k e^{\alpha_{3} l}\right)$,

where $\mathfrak{F}$ depends upon the initial conditions. The expression (4.18) is of the form (4.6) and it follows that, for large enough $k, \widetilde{\Gamma}$ satisfies (4.4). Consequently, steady-state solutions will have the same form for large $k$ whichever approach is employed, as was pointed out by Batchelor (1959).

The analysis for the vector fields is similar, since we can clearly write, at least for large $t, \widetilde{\Psi}_{F}(k)=e^{2 \alpha_{1} t} \widetilde{\Gamma}(k)$, where $\widetilde{\Gamma}(k)$ is the spectrum function of a conserved scalar and is given by (4.18). It follows that for sufficiently large $k$, $\widetilde{\Psi}_{F}(k)$ will be of the form (4.9) and satisfy equation (4.5), and similarly for the G-spectrum. The trouble with the Pearson divergence comes when we try to construct a steady solution by integrating the solution for an instantaneous source of F-variations with respect to the time, for from (4.18)

$$
\tilde{\Psi}_{H^{\prime}}(k) \sim e^{\left(2 \alpha_{1}+\alpha_{3}\right) t} e^{\lambda k^{2} / \alpha_{3}} \mathfrak{F}(0) \rightarrow \infty \text { as } t \rightarrow \infty,
$$

since $2 \alpha_{1}+\alpha_{3}=\alpha_{1}-\alpha_{2}>0$, and $\mathfrak{F}(0)$ is in general non-zero. However, this divergence is related to the fact that in (4.18) the wave-number vector $\mathrm{k}$ was initially $\chi$ and spent a long time near the origin of wave-number space as shown by (4.17). The corresponding Fourier component of $\mathbf{F}$ continued to grow like $e^{2 x_{1} t}$ while the wave-number vector was near $k=0$ and suffered negligible decay due to dissipation. Any cut-off or modification which prevents wave-number vectors from approaching too close to $k=0$ or which does not allow the Fourier 
component to be amplified at an exponential rate while near $k=0$ (as would be appropriate for homogeneous turbulence) can be shown to eliminate the divergence while leaving unaltered the conclusion that (4.5) is satisfied. Thus it would appear again that equation (4.5), which is the Fourier transform of (3.15), may be regarded as a reasonable hypothesis for the spectrum function of the vector fields for large wave-numbers greater than $k_{d}$, while the Pearson divergence which effectively singles out an exponentially growing solution by the behaviour near $k=0$ is not relevant to homogeneous turbulence.

The analysis has been considered here for the case $\alpha_{2}>0$, but a similar argument holds for $\alpha_{2}<0$.

Another argument against the relevance of the Pearson divergence may appropriately be mentioned here. The expression (4.19) appears to hold for all values of $\lambda$ provided $k$ is greater than $k_{d}$. In particular, it holds for $\lambda \gg \nu$, and although $e^{\lambda k^{2 / \alpha_{3}}}$ is then very small since $\alpha_{3}=O\left(-\nu k_{d}^{2}\right)$, it still implies that $\Psi_{F}^{*}(k) \rightarrow \infty$ as $t \rightarrow \infty$ and that therefore $\overline{\mathbf{F}^{2}} \rightarrow \infty$, however large $\lambda / v$ may be. This is inconsistent with the established idea that the growth of $\mathbf{F}$ must be limited by molecular diffusion if the diffusivity $\lambda$ is sufficiently large and, moreoever, with the exact solution $\mathbf{F}=\omega$ which obtains when $\lambda=\nu$. Any argument for the indefinite growth of $\overline{\mathbf{F}^{2}}$ in homogeneous turbulence when $\lambda \ll \nu$ based on the Pearson divergence must therefore also explain why the divergence does not apply when $\lambda \gg v$, or $\lambda=\nu$.

It is worth pointing out that equation (4.4) for $\Gamma(k)$ is also a consequence of a particular assumption about the transfer of spectral density in wave-number space by the non-linear interactions in the equilibrium range. The assumption is that the rate of transfer of spectral density across wave-number magnitude $k$ is $k \Gamma(k) / \tau^{*}$, where $\tau^{*}$ is the characteristic time for the transfer at wave-number $k$. The equation for the conservation of $\Gamma(k)$ then follows as

$$
\frac{\partial \Gamma}{\partial t}=-\frac{\partial}{\partial k}\left(\frac{k \Gamma}{\tau^{*}}\right)-2 \lambda k^{2} \Gamma
$$

(see Corrsin 1961). This equation with $E(k)$, the energy spectrum function, instead of $\Gamma(k)$ has been studied by Ellison (1962), who termed it the modified Obukhov theory. For wave-numbers greater than $k_{d}$, the transfer of spectral density is controlled by the straining motion associated with the small eddies which decreases the scale by bringing level surfaces together, and the appropriate value of $\tau^{*}$ is $-\gamma^{-1}$. Equation (4.20) then reduces to (4.4). For the vector fields, there is also an amplification of the spectral density owing to the stretching of material lines for the $\mathbf{F}$-field or the approach of level surfaces for the $\mathbf{G}$-field. The equation for $\Psi_{F}(k)$ will then be

$$
\frac{\partial \Psi_{F}^{*}}{\partial t}=\frac{2 \Psi_{F}}{\tau^{* *}}-\frac{\partial}{\partial k}\left(\frac{k \Psi_{F}}{\tau^{*}}\right)-2 \lambda k^{2 \Psi_{F}}
$$

where $\tau^{* *}$ is the characteristic time of the amplification due to stretching of material lines. Again for wave-numbers greater than $k_{d}$, the appropriate value of $\tau^{* *}$ is $\alpha^{-1}$, and (4.21) then reduces to (4.5). For the G-field, $\tau^{* *}=\tau^{*}$ since the process which reduces the scale also causes the amplification. 


\section{The structure of the steady state with $\lambda=\nu$}

Our justification of equation (3.15) or equivalently its transform (4.5) is now concluded, and we shall investigate the fine-scale structure as given by their solutions. In physical space, it is actually more convenient (because of the restriction (3.20) on $F$ and $G$ ) to use the equation satisfied by $f$ and $g$ (defined in (3.1) and (3.2)) which is readily found to be

$$
\frac{\partial h}{\partial t}=2 A\left(h+\frac{r}{2 \sigma} \frac{\partial h}{\partial r}\right)+2 \lambda\left(\frac{\partial^{2} h}{\partial r^{2}}+\frac{4}{r} \frac{\partial h}{\partial r}\right)
$$

where $A$ and $\sigma$ have the same meanings as before. The quantity $f$ is the longitudinal covariance $\overline{F_{p} F_{p}^{\prime}}$ and $g$ the lateral covariance $\overline{G_{n} G_{n}^{\prime}}$. At $r=0, f(0)=\frac{1}{3} \overline{\mathbf{F}^{2}}$, $g(0)=\frac{1}{3} \overline{\mathbf{G}^{2}}$. This equation can be put into a dimensionless form by change of variables

$$
z=\frac{A r^{2}}{4 \lambda \sigma}, \quad \tau=\frac{2 A t}{\sigma}, \quad h=e^{-z} y(z, \tau)
$$

and $(5.1)$ then becomes

$$
z y^{\prime \prime}+\left(\frac{5}{2}-z\right) y^{\prime}-\left(\frac{5}{2}-\sigma\right) y=\frac{\partial y}{\partial \tau}
$$

where the primes denote $\partial / \partial z$.

Consider now the G-field in homogeneous turbulence with $\lambda \ll \nu$. We suppose that there is a supply of G-variations with a scale $L$ comparable with that of the energy containing eddies. The straining motion associated with the vorticity acts on larger-scale components and generates small-scale fluctuations of size $l_{r}$. These are amplified as their scale is reduced until the scale becomes sufficiently small for dissipation to destroy them. This decrease of scale and the accompanying initial amplification and eventual decay is exemplified by the solution (3.26) of (3.15) with $\sigma=1$ and $A=-\gamma$. The $\mathbf{G}$-variations of scale $L$ are themselves broken up into smaller scale components by non-linear interactions and if there is no source of fluctuations of length $L$, then they will eventually decay and $\overline{\mathbf{G}}^{2}$ will tend to zero. Now it is usual to assume that there is an ample reservoir of G-variations of large scale for the small-scale variations to be in a state of statistical equilibrium. Then the small-scale structure $\left(r<l_{d}\right.$ or $\left.k>k_{d}\right)$ is given by putting $\partial / \partial t=0$ in (5.1) or (4.5). The regular solution of (5.3) with the righthand side zero is the confluent hypergeometric function ${ }_{1} F_{1}\left(\frac{5}{2}-\sigma ; \frac{5}{2} ; z\right)$. Hence

$$
g(r)=\frac{1}{3} \overline{\mathrm{G}}^{2} e^{-z}{ }_{1} F_{1}\left(\frac{3}{2} ; \frac{5}{2} ; z\right) \quad \text { for } \quad r=\left(-\frac{4 \lambda z}{\gamma}\right)^{\frac{1}{2}}<l_{d}
$$

The corresponding value of $G$ is

$$
G(r)=\overline{\mathbf{G}^{2}} e^{-z}{ }_{1} F_{1}\left(\frac{1}{2} ; \frac{3}{2} ; z\right) .
$$

The spectral density $\Psi_{G}(k)$ is obtained by taking the Fourier transform of (5.5) and is found (Erdélyi, Magnus, Oberhettinger \& Tricomi 1954, p. 74) to be, for $k>k_{d}$,

$$
\Psi_{G}(k)=2 \overline{\mathbf{G}^{2}}\left(\frac{\lambda}{-\gamma}\right) k \exp \left(\frac{\lambda k^{2}}{\gamma}\right)
$$

The constant in $(5,6)$ has been determined by the requirement that it is the transform of (5.5). Note that (4.3) is satisfied by (5.6) although this form of $\Psi_{G}$ 
is not valid for $k<k_{d}$. These results are, of course, all implicit in Batchelor's (1959) results for $\Gamma(k)$, since $\Psi_{G}(k)=k^{2} \Gamma(k)$.

It is to be noted now that the equations possess many other solutions. Thus equation (5.3) also has the solutions

$$
y(z, \tau)=e^{s \tau} F_{1}\left(\frac{5}{2}-\sigma+s ; \frac{5}{2} ; z\right) .
$$

Provided $\frac{5}{2}-\sigma+s \neq-n$, where $n$ is a positive integer or zero, the asymptotic behaviour of $y$ is

$$
y \sim \frac{\left(\frac{3}{2}\right) !}{\left(\frac{3}{2}-\sigma+s\right) !} e^{s \tau} e^{z} z^{s-\sigma} .
$$

The corresponding behaviour of $h$ is (apart from a numerical factor)

$$
h(r) \sim e^{s \tau} r^{2 s-2 \sigma} \text {. }
$$

Thus equation (5.1) has solutions that grow exponentially or decay exponentially in time, all of which moreover are well-behaved and tend algebraically to zero as $r \rightarrow \infty$ (provided $s<\sigma$ for the growing solutions). The analysis of the fine-scale structure itself does not enable us to say which solution is appropriate. This must be determined by the conditions imposed at $r=l_{d}$ (or equivalently at $k=k_{d}$ in wave-number space if we solve (4.5)) where the solution must match that appropriate to the variations on a scale greater than $l_{d}$. For the G-field, we say that the solution should be steady. Note that the values of $s$ cannot be determined by the requirement that the algebraic behaviour of (5.9) should agree with that in the 'convection subrange' (Batchelor 1959) where $g \propto r^{-\frac{4}{3}}$; this gives $s=\frac{1}{3}$ and an exponentially growing solution which is not acceptable.

Let us now consider the F-field with $\lambda \ll \nu$, and suppose that $\sigma<\frac{5}{2}$. Then all solutions of (5.1) which are exponentially small as $r \rightarrow \infty$ eventually decay exponentially with time although there may be (and in general will be) initial amplification. This is again exemplified by the solution (3.26). More generally, let us suppose $\frac{5}{2}-\sigma+s=-n$. Then the confluent hypergeometric function (5.7) reduces to a generalized Laguerre polynomial, and a complete set of solutions of $(5.1)$ is

$$
h=e^{\left(\sigma-\frac{5}{2}-n\right) \tau} e^{-z} L_{n}^{\left(\frac{3}{2}\right)}(z) \quad(n=0,1,2, \ldots),
$$

all of which vanish as $\tau \rightarrow \infty$. Any distribution of $h$ exponentially small at infinity can be expressed as a convergent sum of these functions, showing that there is eventual decay. (Initial amplification at $r=0$ takes place by the higher order terms decaying more rapidly.)

Thus we see that small-scale fluctuations of the $\mathbf{F}$-field behave in a very similar manner to those of the G-field. That is, there will be initial amplification while the scale is comparable with $l_{d}$, but as the scale is reduced to $(\lambda / \nu)^{\frac{1}{2}} l_{d}$, dissipation becomes dominant and destroys the fluctuation. If there is a steady source of such fluctuations, we should expect that a steady state is set up. Is this the appropriate solution for homogeneous turbulence? Certainly there will be fluctuations of scale $l_{d}$ produced by the action of the straining motion on largescale variations if these are present. However, there is the possibility that the turbulent motion on scales larger than $l_{d}$ produces a growing solution. This is how the Pearson divergence is produced, because as we have seen this is associ- 
ated with amplification at zero wave-numbers and this singles out one of the growing solutions of equation (5.1), this equation being valid for an ensemble of infinite straining fields for $r$ not too large, as shown in $\$ \$ 2$ and 3 . But for homogeneous turbulence, we do not expect there to be appreciable amplification on scales larger than $l_{d}$ which, moreover, controls the fine structure. This is suggested by the analogy between $\mathbf{F}$ and the vorticity $\boldsymbol{\omega}$ since on scales greater than $l_{d}$, the viscosity and diffusivity are unimportant and the two vector fields satisfy the same equation. In any event, the fine-scale structure is not unstable in the sense of Batchelor (1952) to the introduction of small amounts of the quantity represented by $\mathbf{F}$, and there is no reason for believing that dissipation does not limit the growth of $\overline{\mathbf{F}^{2}}$ or that the fine-scale structure is unsteady if there is a reservoir of large-scale $\mathbf{F}$-variations.

The covariance for $r<l_{d}$ and the spectral density for $k>k_{d}$ of $\mathbf{F}$ are easily found when conditions are statistically steady. We find from (5.1) and (3.15) that

$$
\begin{gathered}
f(r)=\frac{1}{3} \overline{\mathbf{F}^{2}} e^{-z}{ }_{1} F_{1}\left(\frac{5}{2}-\sigma ; \frac{5}{2} ; z\right) \quad\left(z=\alpha r^{2} / 4 \sigma \lambda\right), \\
F(r)=\overline{\mathbf{F}^{2}} e^{-z}{ }_{1} F_{1}\left(\frac{3}{2}-\sigma ; \frac{3}{2} ; z\right) .
\end{gathered}
$$

The transform of (5.12) gives

$$
\Psi_{F}(k)=\frac{2 \overline{F^{2}}}{(\sigma-1) !}\left(\frac{\sigma \lambda}{\alpha}\right)^{\sigma} k^{2 \sigma-1} \exp \left(-\frac{\lambda \sigma k^{2}}{\alpha}\right)
$$

which, as may readily be verified, satisfies (4.5). The constant of proportionality has again been chosen so that (5.13) is the transform of (5.12); but again the expression satisfies (4.3). The result (5.13) has the same form as that given by Novikov (1962) for the fine-scale vorticity spectrum.

These expressions contain two parameters, $\sigma$ and $\alpha$. The former is expected to be less than one, the latter to be of order $(\epsilon / \nu)^{\frac{1}{2}}$. As mentioned earlier, if we equate $\alpha$ and $\gamma$ to the mean values of the greatest and least rates of strain, we have as estimates $\sigma \div 0 \cdot 8, \alpha \div 0 \cdot 4(\epsilon / \nu)^{\frac{1}{2}}$.

If $\lambda \ll \nu$, the spectrum given by (5.13) behaves like $k^{2 \sigma-1}$ for $k_{d}<k \ll(\nu / \lambda)^{\frac{1}{2}} k_{d}$, and reaches a maximum if $\sigma>\frac{1}{2}$ at the wave-number $(\nu / \lambda)^{\frac{1}{2}} k_{d}$ where most of the dissipation takes place. If $\sigma<\frac{1}{2}$ the amplification due to the straining motion is not strong enough to balance the flux of spectral 'energy' along the wavenumber axis and the spectrum function decays monotonically for $k>k_{d}$.

It remains to discuss the case $\sigma>\frac{5}{2}$. The exponential term in $(5.10)$ is then positive, for the mode $n=0$ at least, and small variations in the fine-scale structure are then in general amplified without limit, and the turbulent motion is unstable to the introduction of a small amount of the quantity represented by $\mathbf{F}$. Indefinite growth in which the convection always dominates the dissipation is therefore a possibility in homogeneous turbulence, although an unlikely one as far as can be seen at present since the evidence points to $\sigma$ being less than one. Any work which throws light on the value of $\sigma$ would clearly be of great value in this connexion. 


\section{The magnetic field in a fluid of large conductivity}

We now examine the consequences of our hypothesis that dissipation limits the growth of $\overline{\mathbf{F}^{2}}$ however small the diffusivity $\lambda$ may be (provided $\sigma<\frac{5}{2}$ ) for the particular case of a magnetic field $\mathbf{H}$ in a good conductor. The amplification of a weak applied magnetic field in a poor or moderate conductor in turbulent motion has been calculated by Golitsyn (1960) and Moffatt (1961). In these cases where $\lambda \gg \nu$, a steady state is possible only if magnetic energy is continuously supplied, and in the absence of permanent electromotive forces the magnetic field eventually decays to zero owing to ohmic dissipation. For a good conductor with $\lambda \ll v$, Batchelor (1950) argued that a seed magnetic field would grow indefinitely until the Lorentz $(j \wedge B)$ force became sufficiently large to modify those properties of the turbulent motion that promote the growth of the magnetic field. A statistically steady state is then achieved that lasts indefinitely provided mechanical energy is fed into the system to maintain the turbulent motion. This would constitute a turbulent dynamo. The present arguments against the idea of indefinite growth throw doubt on the existence of a turbulent dynamo in this sense, but it should be stressed that the present theory in no way contradicts Batchelor's argument that turbulence is an effective amplifier of a magnetic field in a good conductor.

First, let us consider for the case $\lambda \ll v$ the amplification of a weak permanent magnetic field $\mathbf{H}_{\mathbf{0}}$, generated by external electromotive forces, whose scale is that of the energy containing eddies $L$. If ohmic dissipation limits the growth of the magnetic field due to the convection by the turbulence as argued in this paper, a statistically steady state will be set up in which the magnetic energy density is proportional to $\overline{\mathbf{H}^{2}}$. For astrophysical and other applications, it is desirable to obtain an estimate of the ratio $\overline{\mathbf{H}^{2}} / \mathbf{H}_{0}^{2}$. This will be obtained here by the use of arguments presented by Moffatt (1961), with the assumption that the initial field is sufficiently weak for the action of the magnetic field on the turbulence to be neglected. (Since the equation for $\mathbf{H}$ is then linear, the value of this ratio is independent of the magnitude of $\mathbf{H}_{0}$.)

It is supposed that the Reynolds number of the turbulence is sufficiently large for an inertial subrange to exist for wave-numbers $L^{-1} \ll k \ll k_{d}$. It then follows either from the analogy with vorticity or from an argument using the vector potential A (Moffatt 1961) that in the inertial subrange, the spectrum function is given by

$$
\Psi_{H}(k) \approx \frac{\chi_{A}}{\epsilon^{\frac{1}{3}}} k^{\frac{1}{3}}
$$

where $\chi_{A}$ can be identified with the rate of destruction of $\overline{\mathbf{A}^{2}}$-stuff by conduction. (Moffatt in fact supposed that $\lambda \gg \nu$ and considered that part of the inertial subrange in which $k \ll\left(\epsilon / \lambda^{3}\right)^{\frac{1}{4}}$, but his argument if valid clearly applies to the whole inertial subrange if $\lambda \leqslant \nu$.) We now assume that $(6.1)$ is of the same order of magnitude as (5.13) for wave-numbers of order $\left(\epsilon / v^{3}\right)^{t}$. It follows that

$$
\overline{\mathbf{H}^{2}}\left(\frac{\lambda}{\nu}\right)^{\sigma-1} \approx \frac{\chi_{A}}{\lambda}
$$


factors of order unity being neglected. (Note that if $\sigma=\frac{2}{3}$, which is a possible value, (6.1) and (5.13) have the same dependence upon $k$ for wave-numbers comparable with $\left(\epsilon / \nu^{3}\right)^{\frac{1}{1}}$.)

Without turbulence, only the large-scale distribution is present, with meansquare value of approximately

$$
\mathbf{H}_{\mathbf{0}}^{2} \approx \int_{0}^{L^{-1}} \Psi_{H}(k) d k \approx L^{-1} \Psi_{H}\left(L^{-1}\right) .
$$

We suppose further than (6.1) holds, at least in order of magnitude, down to $k=L^{-1}$, so that

$$
\Psi_{H}\left(L^{-1}\right) \approx \frac{x_{A}}{\epsilon^{\frac{1}{3}}} L^{-\frac{1}{2}}
$$

Then using (6.2) and the relation $\epsilon \approx\left(\overline{\mathbf{u}^{2}}\right)^{\frac{3}{2}} / L$, we obtain

$$
\overline{\mathbf{H}^{2}} \approx\left(\frac{\nu}{\lambda}\right)^{\sigma} R \mathbf{H}_{0}^{2}=\left(\frac{\lambda}{\nu}\right)^{1-\sigma} R_{M} \mathbf{H}_{0}^{2}
$$

where $R$ is the Reynolds number $\left(\overline{u^{2}}\right)^{\frac{1}{2}} L / v$, and $R_{M}$ is the magnetic Reynolds number.

Thus the magnetic energy is amplified to a considerable extent by the turbulence since both $R$ and $\nu / \lambda$ will be large. It is interesting to note that Moffatt found $\overline{\mathbf{H}^{2}} \approx R_{M} \mathbf{H}_{0}^{2}$ for $\nu / \lambda \ll 1$ but $R_{M} \gg 1$ (factors of order unity being neglected). The results for the two cases join smoothly at $\nu=\lambda$.

Again following Moffatt, we can estimate the increased loss of energy by Joule heating. Thus if

$$
D_{0}=\lambda \int_{0}^{L^{-1}} k^{2} \Psi_{H}(k) d k \approx \lambda L^{-3} \Psi_{H}\left(L^{-1}\right)
$$

is the dissipation in the absence of turbulence, and

$$
D=\lambda \int_{0}^{\infty} k^{2} \Psi_{H}(k) d k \approx \alpha \overline{\mathbf{H}^{2}}
$$

is the dissipation when the flow is turbulent,

$$
D \approx R^{\frac{5}{2}}\left(\frac{\nu}{\lambda}\right)^{\sigma+1} D_{0}
$$

The result for a fluid with $\nu \ll \lambda$ is $D \approx R_{M}^{5} D_{0}$.

The condition that Lorentz forces should be negligible is that the mean magnetic energy $\overline{\mathbf{H}^{2}} / 8 \pi$ (c.g.s. units) should be small compared with the kinetic energy density associated with the small-scale straining motion $\rho(\epsilon v)^{\frac{1}{2}}$. That is

$$
\mathbf{H}_{\mathbf{0}}^{2} \ll 8 \pi \rho \overline{\mathbf{u}^{2}} R^{-\frac{3}{2}}(\lambda / \nu)^{\sigma} .
$$

For larger values of $\mathbf{H}_{0}^{2}$, the growth of magnetic energy will be inhibited by the action of the Lorentz forces on the turbulence and is unlikely to reach the level given by (6.5). Indeed, in most practical cases it seems that the limitation of the growth of magnetic energy by ohmic dissipation will be unimportant and that the equilibrium value (in the presence of a permanent applied field) will be 
determined by the back reaction of the magnetic field on the turbulence. The present analysis is then invalid, as it has been assumed that $\alpha, \gamma$ and $\sigma$ have the values appropriate to turbulence in the absence of external forces. Nevertheless, our equations for the fine-scale structure may still be valid with the proviso that the quantities $\alpha$ and $\gamma$, that measure the convection effects, depend upon the magnetic field. The tension in the lines of force will resist the stretching by the turbulence, so that $\alpha$ may be expected to decrease as $\overline{\mathbf{H}^{2}}$ increases until it reaches a value for which no further amplification of the magnetic field ocurs. Since $\alpha$ is a property of the small eddies, this may imply that the equilibrium level is determined by Batchelor's (1950) criterion of equipartition of energy between the magnetic field and the motion of the small eddies, and not by the Biermann \& Schluiter (1951) criterion of equipartition of energy between the magnetic field and the energy containing eddies of the turbulence, but it is hoped to discuss this matter is more detail in a later paper.

To come now to the question of the turbulent dynamo when $\lambda \ll \nu$, suppose that a weak seed field of length scale $L$ is imposed by a temporary electromotive force and there is no other source of electromagnetic energy. The small eddies then generate small-scale fluctuations whose amplification and eventual decay is described statistically by our equations. It follows from the equations (e.g. the particular solution (3.26)) that a fluctuation lasts for a finite time of order $-\gamma^{-1} \log (\nu / \lambda)$. Thus the magnetic field will be amplified only so long as the large-scale components persist. Now if there is no external source of magnetic energy, these will be gradually destroyed by the non-linear interactions with themselves, $\uparrow$ and they may be expected to persist only for a time $T$ comparable with the time-scale of the energy containing eddies; $T \approx \epsilon / \overline{\mathbf{u}^{2}}$. After this time, no more small-scale fluctuations are generated and the magnetic field decays. Thus the total magnetic field, according to this picture, does not exist for a time larger than the greater of $T$ or $-\gamma^{-1} \log (\nu / \lambda)$. (Note that $T \gg-\gamma^{-1}$.) In other words, the turbulent dynamo does not exist. The amplification will nevertheless be large. Indeed, if $T \gg-\gamma^{-1} \log (\nu / \lambda)$, the small-scale structure will be in a state of quasi-equilibrium and the amplification is given by (6.5), provided the Lorentz forces are negligible.

In practice, the Lorentz forces are unlikely to be negligible because of the large amplification and they will restrict the amplification as for the case of a permanent applied field. There seems to be no way in which the Lorentz forces can generate small-scale fluctuations of magnetic field (this would require an extra term in the equations for the correlations which does not seem to exist), and the argument that the turbulent dynamo does not exist in the strict sense would appear to be unaffected. Nevertheless, an effective dynamo exists for a time $T$. Moreover, the idea of this paper that the magnetic energy is generated at wave-number $k_{d}$ and transferred to wave-number $(\nu / \lambda)^{\frac{1}{2}} k_{d}$, where it is destroyed by ohmic dissipation, the amplification and destruction of magnetic energy therefore being a property of the small eddies, seems to support Bat-

$\dagger$ This process would be represented in wave-number space by a cascade process which transfers spectral density along the wave-number axis towards larger wave-numbers with a time scale $T$. 
chelor's hypothesis that the strength of the effective dynamo is determined by a balance between the magnetic energy and the kinetic energy of the small eddies.

\section{Appendix. Application to the vorticity}

Since the vorticity field $\omega$ satisfies equation (1.2) with $\lambda=v$, a natural suggestion is that the vorticity covariance

$$
W(r)=\overline{\omega_{i}}(\mathbf{x}) \omega_{i}(\mathbf{x}+\mathbf{r})
$$

may satisfy equation (3.15) with appropriate values of $\alpha$ and $\sigma$ for values of $r$ less than $l_{d}$. There seem to be two main objections to this postulate. First, the vorticity is not a dynamically passive quantity and secondly the 'Prandtl number' is unity instead of being large. These objections throw doubt on the argument for (3.15) based on the orientation of 'lines of force' and level surfaces, but they do not prove it wrong, especially since the argument works when the fluid rotates relative to the principal axes of rate of strain, rotation of the fluid being one of the dynamical effects of vorticity. Moreoever, the argument for (3.15) based on the form of the exact equation and dimensional considerations would seem to be unaffected with the proviso that the parameters $\alpha$ and $\sigma$ may have values different from those for a dynamically passive quantity with large Prandtl number. At the very least, the consequences of the postulate as a working hypothesis seem worth examining.

For turbulence with large Reynolds number, the fine-scale motion is in statistical equilibrium and the steady solution of (3.15) is the appropriate one. This is completely determined by the condition that $W(0)$ is finite and equal to $\epsilon / v$. Thus (see (5.12)) for $r<l_{d}$

$$
W(r)=\frac{\epsilon}{\nu} \exp \left(\frac{-\alpha r^{2}}{4 \sigma \nu}\right){ }_{1} F_{1}\left(\frac{3}{2}-\sigma ; \frac{3}{2} ; \frac{\alpha r^{2}}{4 \sigma \nu}\right) .
$$

If $E(k)$ denotes the energy spectrum function, the vorticity spectrum function is $2 k^{2} E(k)=\Omega(k)$, say, where $\Omega(k)$ is related to $W(r)$ by (4.1). It follows (see Erdélyi et al. 1954, p. 74, and equation (5.13)) that

$$
E(k)=\frac{\epsilon}{\nu}\left(\frac{\nu \sigma}{\alpha}\right)^{\sigma} \frac{k^{2 \sigma-3}}{(\sigma-1) !} \exp \left(\frac{-\nu \sigma k^{2}}{\alpha}\right) .
$$

The range of validity of this expression is not altogether certain. Mathematically, we should expect it to hold for $k \gg k_{d}$, but physically it may be expected to hold for wave-numbers larger than those at which most of the vorticity is found. The vorticity spectrum is found experimentally to have a maximum for many different circumstances at wave-numbers slightly less than $0 \cdot 2 k_{d}$ (see Grant, Stewart \& Moilliet 1962). It is to be noted that (A 3) satisfies the exact relation

$$
\epsilon=2 v \int_{0}^{\infty} k^{2} E(k) d k
$$

and there would be no inconsistencies if (A 3 ) held down to $0 \cdot 2 k_{d}$. 
The quantity $\alpha$ is related to the skewness factor $S$ of the turbulence defined by

From the exact relation

$$
S=\overline{\left(\frac{\partial u_{1}}{\partial x_{1}}\right)^{3}} /\left[\left(\overline{\left.\frac{\partial u_{1}}{\partial x_{1}}\right)^{2}}\right]^{\frac{3}{2}}\right.
$$

$$
\frac{1}{2} \frac{\partial \overline{\omega^{2}}}{\partial t}=\overline{\omega_{i} \omega_{j} \frac{\partial u_{i}}{\partial x_{j}}-\nu\left(\frac{\partial \omega_{i}}{\partial x_{j}}\right)^{2}},
$$

it follows (see Batchelor \& Townsend 1956) that

$$
\alpha=\frac{\overline{\omega_{i} \omega_{j}\left(\partial u_{i} / \partial x_{j}\right)}}{\overline{\omega^{2}}}=-\frac{7}{6 \sqrt{15}}\left(\frac{\epsilon}{v}\right)^{\frac{1}{2}} S
$$

The measured values of $S$ lie between -0.3 and -0.5 , so that $\alpha$ lies between $0 \cdot 09(\epsilon / \nu)^{\frac{1}{2}}$ and $0 \cdot 15(\epsilon / \nu)^{\frac{1}{2}}+\uparrow$ The value of $\sigma$ can be estimated from the measured value of

$$
\begin{aligned}
\overline{\left(\frac{\partial^{3} u_{1}}{\partial x_{1}^{3}}\right)^{2}}\left(\overline{\left.\frac{\partial u_{1}}{\partial x_{1}}\right)^{2}} /\left[\overline{\left(\frac{\partial^{2} u_{1}}{\partial x_{1}^{2}}\right)^{2}}\right]^{2}\right. & =\frac{165}{243} \frac{W(0)}{\left[W^{\prime \prime}(0)\right]^{2}} \\
& =\frac{35}{27} \frac{\sigma+1}{\sigma} \text { from (A 2). }
\end{aligned}
$$

Measured values of this quantity (Batchelor \& Townsend 1949), indicate that it is rougly equal to $4 \cdot 5$ for large Reynolds number, which gives $\sigma \approx 0 \cdot 4$. This value is consistent with the belief that $\sigma$ is generally less than unity.

An expression of the form (A 3) was derived by Novikov (1962) by an extension of Batchelor's (1959) analysis in wave-number space, but the present approach seems superior in that it allows the parameters to be related exactly to measurable quantities, apart from the theoretical objections to the analysis in wave-number space mentioned earlier.

For the purpose of comparison with experiment, it is necessary to evaluate the longitudinal spectrum function

$$
\phi(k)=\int_{k}^{\infty}\left(1-\frac{k^{2}}{\kappa^{2}}\right) \frac{E(\kappa)}{\kappa} d \kappa
$$

since this is the quantity measured experimentally. (The definition (A 8) gives a $\phi$ twice that defined by Batchelor 1953.) The substitution of (A 3) into (A 8) gives, after change of variable,

$$
\phi(k)=\epsilon^{\frac{\pi}{4}} \nu^{\frac{5}{4}} c^{-\frac{3}{2}} \sigma n^{2 \sigma-3} J_{\sigma}(n), \S
$$

where

$$
J_{\sigma}(n)=\frac{1}{\sigma !} \int_{0}^{1} x^{2-2 \sigma}\left(1-x^{2}\right) \exp \left(-n^{2} / x^{2}\right) d x
$$

and

$$
n=c^{-\frac{1}{2} k / k_{d}}, \quad c=\alpha \nu^{\frac{1}{2}} / \sigma \epsilon^{\frac{1}{2}}=-\frac{7}{90} \sqrt{ } 15 S / \sigma .
$$

$\dagger$ If the principal axes of rate of strain did not rotate relative to the fluid, it might be expected that $\alpha \approx \overline{\alpha_{1}}$, the mean value of the greatest principal rate of strain. Estimates of $\overline{\alpha_{1}}$ are about $0 \cdot 4(\epsilon / v)^{\frac{1}{2}}$.

$\ddagger$ I am indebted to Dr A. A. Townsend for this observation.

$\S J_{\sigma}$ is not to be confused with the Bessel function of order $\sigma$. 
The expression (A 9) is not very different in form from that given by Townsend $(1951 a)$, which can be written in the present notation

$$
\phi_{T}(k)=D \epsilon^{\frac{1}{2}} \nu^{\frac{5}{2}}\left(\frac{k}{k_{d}}\right)^{-2} J_{1}\left(\frac{2^{\frac{1}{2}} k}{k_{d}}\right)
$$

where $D$ is an arbitrary parameter.

Townsend (1951 $a$ ) compared $k^{6} \phi_{T}(k)$ with experimental measurements and found very good agreement for $k / k_{d}>0.2$ if $D$ is put equal to about 0.4 (the value of $D$ is not stated by Townsend but this seems to be about the value used). The reason for multiplying by $k^{6}$ was to bring out that part of the spectrum determined by the smallest eddies. From (A 9),

$$
\left(\frac{k}{k_{d}}\right)^{6} \frac{\phi(k)}{\epsilon^{\frac{1}{4} \nu^{\frac{5}{4}}}}=c^{\frac{3}{2}} \sigma n^{2 \sigma+3} J_{\sigma}(n)
$$

In figure 1 , we have plotted $k^{6} \phi$ for the particular values $S=-0 \cdot 4, \sigma=0 \cdot 4$ as suggested by observations. The Townsend curve, which is close to Townsend's experimental values, is also shown. It can be seen that the present theory gives a curve of the same shape but the amplitude is less and the maximum occurs at a smaller value of $k$. Further measurements of $\phi(k)$ for very large wave-numbers can be found in the data of Grant et al. (1962). However, these show that $k^{6} \phi$ increases monotonically with $k$ for each experimental run, and there is no sign of a maximum. Moreover, the results show considerable scatter for very large wave-numbers and it is likely that the measurements for very large wavenumbers were seriously affected by noise. Townsend's measurements are open to the same objection.

However, Dr Townsend (private communication) has pointed out that the discrepancy may be partly due to the Reynolds numbers of the experiments not being sufficiently high for the small eddies to be in equilibrium, with production of vorticity balancing the dissipation so that the left-hand side of (A 5) is negligible as assumed in the theory. Unfortunately, this was not so in the experiments. Dr Townsend has commented further that if the skewness factor is calculated by equating $\overline{\omega_{i} \omega_{j}\left(\partial u_{i} / \partial x_{j}\right)}$ to $\nu\left(\overline{\left.\partial \omega_{i} / \partial x_{j}\right)^{2}}\right.$, the second term being calculated from the measured spectra, then one obtains $S=-0.6$ against the measured value of $-0 \cdot 4$. It is interesting that the values $S=-0 \cdot 6, \sigma=0 \cdot 4$ bring the theoretical curve (A 13) much closer to the Townsend curve. Alternatively, we may take account of the lack of equilibrium in an approximate manner by replacing the left-hand side of (3.15) by the difference between $\left.\overline{2 \omega_{i} \omega_{j}\left(\partial u_{i} / \partial x_{j}\right.}\right)$ and $2 v\left(\overline{\left.\partial \omega_{i} / \partial x_{j}\right)^{2}}\right.$ and then ignoring the time dependence. According to Batchelor \& Townsend (1948), the ratio of these terms is $\left[1+60 /\left(7 R_{\lambda} S\right)\right]^{-1}$, and it can then be deduced that

$$
W(r)=\left(1+\frac{60}{7 R_{\lambda} S}\right) \frac{\epsilon}{\nu} \exp \left(\frac{-\alpha r^{2}}{4 \sigma \nu}\right){ }_{1} F_{1}\left(\frac{3}{2}-\sigma ; \frac{3}{2} ; \frac{\alpha r^{2}}{4 \sigma \nu}\right)-\frac{60}{7 R_{\lambda} S}
$$

It follows that the corresponding spectrum function for small $k$ is (A 9) multiplied by $1+60 /\left(7 R_{\lambda} S\right)$, and multiplying by this factor brings the present theoretical curve into much better agreement with the data given by Townsend. 
Further support for Townsend's comment is provided by some unpublished measurements by Kistler \& Vrebalovich which are also shown in the figure. These were taken in a wind tunnel at the Jet Propulsion Laboratory at values of $R_{\lambda}$ between 230 and 750 which seems to have been large enough for the small eddies to be in equilibrium. The data do not extend to large enough wavenumbers for the existence of a maximum for $k^{6} \phi$ to be conclusively shown, but it is interesting that they agree better with the present theory assuming equilibrium than with the Townsend expression.

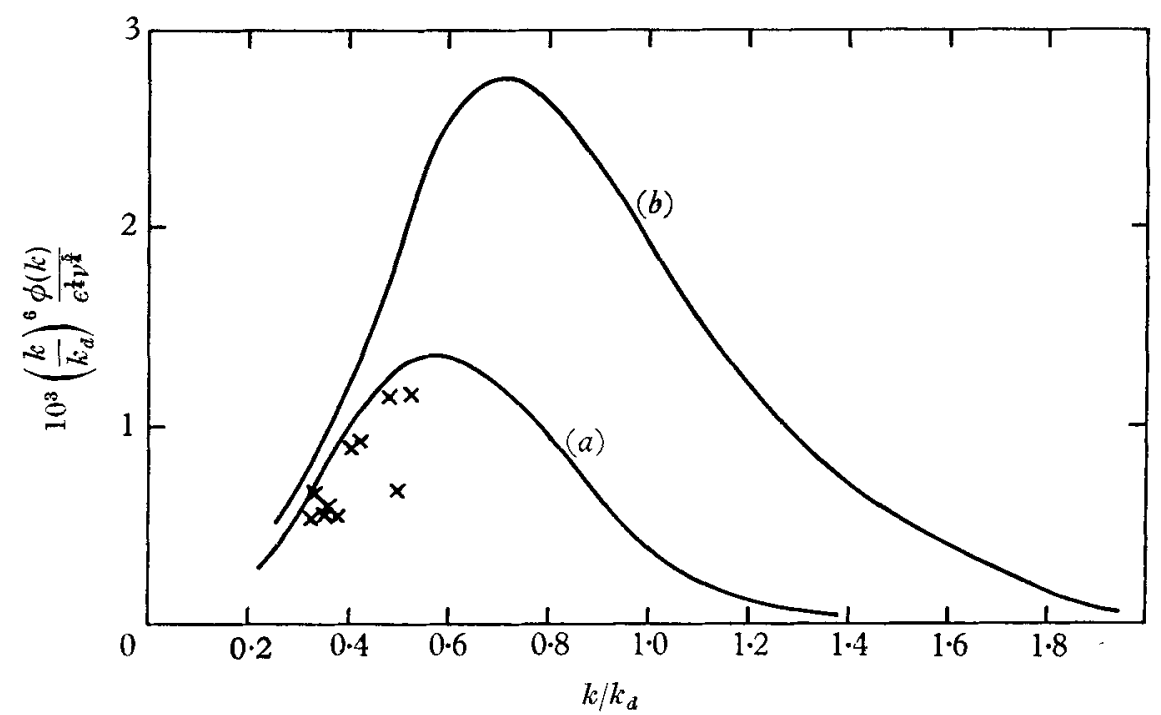

Figure 1. Comparison of present theory with that of Townsend (1951 $a$ ). Curve $(a)$ : dimensionless plot of $k^{6} \phi(k)$ with $\sigma=0 \cdot 4, S=-0.4$. Curve $(b)$ : Townsend's result for a random array of vortex sheets. $x$ : unpublished data by Kistler \& Vrebalovich.

The assumption that the vorticity covariance satisfies (3.15) has some interesting consequences for the transfer of energy between Fourier components of large wave-number. For it is a consequence of $(3.15)$ that $\Omega(k)$ satisfies $(4.5)$, from which it follows that $E(k)$ satisfies

$$
\frac{\partial E}{\partial t}=2 \alpha\left(1-\sigma^{-1}\right) E-\frac{\alpha}{\sigma} \frac{\partial}{\partial k}(k E)-2 \lambda k^{2} E
$$

for $k \gg k_{d}$. The second term on the right-hand side describes the transfer of energy in wave-number space according in effect to the modified Obukhov hypothesis (Ellison 1962). The last term is the ordinary viscous decay, but the first term is new and represents an extraction of energy (if $\sigma<1$ ) from the very small eddies by the non-linear interactions. Since these interactions conserve total energy, there must be a corresponding input of energy into eddies of wavenumber of about or less than $k_{d}$. This input cannot extend into the inertial subrange as it would then contradict the $k^{-\frac{5}{3}}$ behaviour. The physical mechanism by which energy is transferred into the small eddies from the very small eddies is not clear, but if the present theory is at all correct, then it implies that the 
well-known theories of Heisenberg, Obukhov and Kovásznay for transfer of energy in the viscous subrange are in error as they do not allow for any such transfer of energy.

It is a pleasure to record my gratitude to Dr G. K. Batchelor for the stimulation I have received from discussion and argument with him on the subject of this paper. I also wish to thank Dr H. K. Moffatt for many valuable comments. I am also indebted to the Jet Propulsion Laboratory, California Institute of Technology, for their hospitality while the final draft of this paper was prepared, for help with the computations, and for bringing to my notice the unpublished data by Kistler and Vrebalovich.

\section{REFERENCES}

Arexandrou, N. 1963 Ph.D. Thesis, University of London.

Batchelor, G. K. 1950 Proc. Roy. Soc. A, 201, 405.

Batchelor, G. K. 1952 Proc. Roy. Soc. A, 213, 349.

BAtchelor, G. K. 1953 The Theory of Homogeneous Turbulence. Cambridge University Press.

Batchelor, G. K. 1959 J. Fluid Mech. 5, 113.

Batchelor, G. K. \& Townsend, A. A. 1948 Proc. Roy. Soc. A, 193, 539.

Batchelor, G. K. \& Townsend, A. A. 1949 Proc. Roy. Soc. A, 199, 238.

Batchelor, G. K. \& Townsend, A. A. 1956 Surveys in Mechanics. (Eds. Batchelor and Davies.) Cambridge University Press.

Biermann, L. \& Schlüter, A. 1951 Phys. Rev. 82, 863.

Corrsin, S. 1961 J. Fluid Mech. 11, 407.

Eulison, T. H. 1962 Mecanique de la Turbulence, p. 113. Editions du Centre National de la Recherche Scientifique, Paris.

Erdélyi, A., Magnus, W., Oberhettinger, F. \& Tricomt, F. G. 1954 Tables of Integral Transforms, Vol. 1. New York: McGraw-Hill.

Golitsyn, G. S. 1960 Soviet Physics Doklady, 5, 536.

Grant, H. L., Stewart, R. W. \& Mollinet, A. 1962 J. Fluid Mech. 12, 241.

MoffatT, K. 1961 J. Fluid Mech. 11, 625.

Novikov, E. A. 1962 Soviet Physics Doklady, 6, 571.

Prarson, J. R. A. 1959 J. Fluid Mech. 5, 274.

Townsend, A. A. 1951 a Proc. Roy. Soc. A, 208, 534.

Townsend, A. A. 1951 b Proc. Roy. Soc. A, 209, 418. 